\title{
Centromere positions in chicken and Japanese quail chromosomes: de novo centromere formation versus pericentric inversions
}

\author{
Anna Zlotina • Svetlana Galkina • Alla Krasikova • \\ Richard P. M. A. Crooijmans • Martien A. M. Groenen • \\ Elena Gaginskaya $\cdot$ Svetlana Deryusheva
}

Published online: 10 November 2012

(C) Springer Science+Business Media Dordrecht 2012

\begin{abstract}
Chicken (Gallus gallus domesticus, GGA) and Japanese quail (Coturnix coturnix japonica, CCO) karyotypes are very similar. They have identical chromosome number $(2 n=78)$ and show a high degree of synteny. Centromere positions on the majority of orthologous chromosomes are different in these two species. To explore the nature of this divergence, we used highresolution comparative fluorescent in situ hybridization mapping on giant lampbrush chromosomes (LBCs) from growing oocytes. We applied 41 BAC clones specific for GGA1, 2, 3, 11, 12, 13, 14, and 15 to
\end{abstract}

Responsible Editor: Herbert Macgregor.

Electronic supplementary material The online version of this article (doi:10.1007/s10577-012-9319-7) contains supplementary material, which is available to authorized users.

A. Zlotina $(\bowtie) \cdot$ S. Galkina $\cdot$ A. Krasikova •

E. Gaginskaya $(\bowtie) \cdot S$. Deryusheva

Saint-Petersburg State University,

Oranienbaumskoe sh., 2, Stary Peterhof,

Saint-Petersburg 198504, Russia

e-mail: anna-zlotina@yandex.ru

e-mail:spbchromas@gmail.com

R. P. M. A. Crooijmans · M. A. M. Groenen

Animal Breeding and Genomics Centre,

Wageningen University,

Wageningen, The Netherlands

Present Address:

S. Deryusheva

Department of Embryology,

Carnegie Institution for Science,

Baltimore, MD, USA chicken and quail LBCs. This approach allowed us to rule out a pericentric inversion earlier proposed to explain the difference between GGA1 and CCO1. In addition to a well-established large-scale pericentric inversion that discriminates GGA2 and $\mathrm{CCO} 2$, we identified another, smaller one in the large inverted region. For the first time, we described in detail inversions that distinguish GGA3 from $\mathrm{CCO} 3$ and GGA11 from CCO11. Despite the newly identified and confirmed inversions, our data suggest that, in chicken and Japanese quail, the difference in centromere positions is not mainly caused by pericentric inversions but is instead due to centromere repositioning events and the formation of new centromeres. We also consider the formation of short arms of quail microchromosomes by heterochromatin accumulation as a third scenario that could explain the discrepancy in centromeric indexes.

Keywords chicken - evolutionary new centromere · fluorescent in situ hybridization (FISH) - Japanese quail . lampbrush chromosome (LBC) · pericentric inversion
Abbreviations
BAC
Bacterial artificial chromosome
$\mathrm{CCO}$
Japanese quail (Coturnix coturnix
japonica) chromosome
CENP-A Centromere protein-A
CNM
Chicken erythrocyte nuclear membrane repeat
DAPI 4',6-Diamidino-2-phenylindole 
DOP-PCR Degenerate oligonucleotide primed polymerase chain reaction

ENC Evolutionary new centromere

FISH Fluorescence in situ hybridization

GGA Chicken (Gallus gallus domesticus) chromosome

LBC Lampbrush chromosome

$\mathrm{Mb} \quad$ Megabase

\section{Introduction}

The domestic chicken (Gallus gallus domesticus, GGA) and Japanese quail (Coturnix coturnix japoni$c a, \mathrm{CCO}$ ), two representative species of the order Galliformes with a significant agricultural value, diverged about 35 million years ago (van Tuinen and Dyke 2004). These species have typical avian karyotypes that consist of several pairs of relatively large macrochromosomes (chromosomes 1-10 and a pair of sex chromosomes, $\mathrm{ZW}$ in females and $\mathrm{ZZ}$ in males) and numerous tiny microchromosomes. The total number of chromosomes is the same in both species $(2 n=78)$. Furthermore, a high degree of syntenic conservation has been demonstrated using different approaches, such as comparative gene mapping (Shibusawa et al. 2001; Schmid et al. 2005; Galkina et al. 2006; Kayang et al. 2006), chromosome painting (Schmid et al. 2000; Guttenbach et al. 2003), and linkage analysis (Kayang et al. 2006; Sasazaki et al. 2006). The conservation of both macro- and microchromosomes suggests the absence of interchromosomal rearrangements accompanying the divergence of the chicken and Japanese quail. At the same time, the morphology of orthologous chromosomes is different in these two species. The majority of chicken microchromosomes are acrocentric whereas quail microchromosomes are primarily submetacentric (Kaelbling and Fechheimer 1983; Calderón and Pigozzi 2006; Krasikova et al. 2006, 2009). Centromere positions on some macrochromosomes are also different (Ryttman and Tegelstrom 1981; Shibusawa et al. 2001, 2004). First attempts to explain the discrepancy were done using high-resolution G-banding of metaphase chromosomes (Ryttman and Tegelstrom 1981; Sasaki 1981; Stock and Bunch 1982). It is worth noting that the extremely small size of microchromosomes, the smallest one containing only $3.4 \mathrm{Mb}$ of DNA (Pichugin et al.
2001), makes it impossible to perform differential staining of microchromosomes at the metaphase stage. The comparative analysis of macrochromosome banding patterns revealed mismatches of certain chromosomal blocks between chicken and quail chromosomes 1, 2, 4, 6 , and Z. Based on these observations, pericentric inversions were proposed as the most apparent mechanism of intrachromosomal rearrangements that changes centromere positions (Ryttman and Tegelstrom 1981; Sasaki 1981; Stock and Bunch 1982).

A new wave of comparative cytogenetic analysis of chicken and quail karyotypes was initiated when several libraries of chicken genome cloned fragments such as those cloned in bacterial artificial chromosomes (BACs) became available (e.g., Crooijmans et al. 2000, reviewed in Schmid et al. 2005), and integrated resources for chicken genome assembly were released (http://www.ncbi.nlm.nih.gov/genome/111, http://useast.ensembl.org/Gallus_gallus/Info/Index, http://genome.ucsc.edu). Fluorescent in situ hybridization (FISH) of the chicken-specific probes on chicken and quail metaphase chromosomes first confirmed the predicted pericentric inversions on chromosomes 1, 2, and 4 (Shibusawa et al. 2001; Schmid et al. 2005; Kayang et al. 2006). However, precise FISH mapping is tricky in birds, even on macrochromosomes. In some cases, signals for two closely located probes cannot be resolved, or they can even show an order opposite to the known one according to genome sequence assembly (Galkina et al. 2006).

FISH on giant lampbrush chromosomes (LBCs) isolated from growing oocytes (for more information about LBCs, see reviews Callan 1986; Morgan 2002; Gaginskaya et al. 2009; this issue) offers opportunities for gene mapping at a much higher resolution than would ever be possible with metaphase chromosomes (Ogawa et al. 1997; Solovei et al. 1998; Galkina et al. 2006; Krasikova et al. 2006; Deryusheva et al. 2007; Solinhac et al. 2010; Zlotina et al. 2010). When chicken BAC probes were applied to quail LBCs, despite the differences in centromere positions, the gene order identified by FISH on CCO4 was the same as the gene order on GGA4 (Galkina et al. 2006). Thus, the high-resolution mapping did not confirm the pericentric inversion proposed earlier based on G-banding analysis and comparative FISH-mapping on metaphase chromosomes. What other chromosomal rearrangements that might have occurred during karyotype evolution in Galliformes could be resolved using 
the FISH on lampbrush chromosomes as an investigative procedure? On the other hand, why should we still use time-consuming methods like comparative FISH mapping on LBCs when next-generation sequencing approaches are opening up new opportunities for genome-wide analysis? Even when deep sequencing analysis becomes a routine technique in many laboratories, sequence alignment in the regions with highly repetitive sequences is challenging and in many cases unreliable. Therefore, the regions of repetitive sequences are underrepresented in sequence assemblies even for well-annotated genomes. At the same time, some functionally important chromosomal domains, such as centromeres and telomeres, mostly consist of the repetitive DNA. LBCs provide a good system for highresolution FISH mapping when well-resolved hybridization signals can be assigned precisely to particular chromomeres and landmarks on detailed cytological maps constructed for individual chromosomes (Galkina et al. 2006; Krasikova et al. 2006; Deryusheva et al. 2007; Solinhac et al. 2010; Zlotina et al. 2010). Another major advantage of using LBCs is the possibility of identifying centromere position regardless of whether centromere-specific sequences are known. Previously, we have shown that cohesinenriched spherical structures detectable on LBCs by immunostaining with antibodies against core proteins of the cohesin complex are reliable markers for centromere positioning (Krasikova et al. 2006; Deryusheva et al. 2007; Zlotina et al. 2010). One can argue that immunostaining for cohesin-complex proteins that we use to determine centromere position on LBCs is not a canonical centromere identifier. Recent discovery of Centromere protein A (CENP-A)-associated sequences in chicken (Shang et al. 2010) provides an additional support for our centromere mapping approach. The sequences associated with a well-established centromere marker, CENP-A, are assigned to those positions on chicken LBCs that we identified as centromere regions according to cohesin-enriched granule mapping (Krasikova et al. 2012).

In the present work, we use a combination of centromere immunodetection and high-resolution comparative FISH mapping of chicken BAC clones on chicken and quail LBCs to study in detail intrachromosomal rearrangements that must have accompanied genomic divergence during speciation. We focus here on macrochromosomes 1, 2, and 3 and the largest microchromosomes 11, 12, 13, 14, and 15. Our new and earlier obtained data suggest that pericentric inversions are not a predominant mechanism of chromosome morphology alteration in chicken and Japanese quail karyotype evolution. The differences in centromere positions on orthologous chromosomes in these two species are rather the result of new centromere formation and/or the accumulation of heterochromatin that forms prominent short arms of quail submetacentric microchromosomes.

\section{Materials and methods}

\section{Chromosome preparation}

Chicken and Japanese quail LBCs were isolated manually from oocytes of $0.5-1.5 \mathrm{~mm}$ diameter according to standard protocols (http://projects.exeter.ac.uk/ lampbrush/protocols.htm). Preparations were dehydrated in $96 \%$ ethanol and air-dried before FISH, whereas before immunostaining preparations were kept in $70 \%$ ethanol.

\section{Immunofluorescent staining}

To detect centromere positions on chicken and quail LBCs, preparations were stained with rabbit polyclonal antibodies K828 or K854 against STAG2 and $\operatorname{Rad} 21$ cohesin subunits, correspondingly, as previously described (Krasikova et al. 2005). After image acquisition, slides were washed in $4 \times \mathrm{SSC}, 0.1 \%$ Tween 20 at $42{ }^{\circ} \mathrm{C}$, dehydrated in $96 \%$ ethanol, air-dried, and then used for FISH.

\section{FISH}

BAC clones from the Wageningen chicken BAC library (Crooijmans et al. 2000, http://www.bioinformatics.nl/ gbrowse/cgi-bin/gbrowse) were selected based on the genomic positions assigned for corresponding markers. The selected BAC clones are listed in Table 1. Chicken whole chromosome painting probe F12 specific for an individual microchromosome pair (Griffin et al. 1999) and a chromosome 13 painting probe (Masabanda et al. 2004) were kindly provided by Dr. F. A. Habermann (Ludwig-Maximilians-University of Munich, Germany) and Prof. D. K. Griffin (School of Biosciences, University of Kent, Canterbury, UK), respectively. DNA of BAC clones and chromosome paints were 
Table 1 BAC clones mapped to chicken and Japanese quail LBCs

\begin{tabular}{lll}
\hline BAC clone name & $\begin{array}{l}\text { Genetic marker } \\
\text { or GenBank } \\
\text { accession number }\end{array}$ & $\begin{array}{l}\text { Chicken } \\
\text { chromosome } \\
\text { location }^{\mathrm{a}}, \mathrm{Mb}\end{array}$ \\
\hline
\end{tabular}

Chromosome 1

WAG31B10

WAG13E20

LEI0146

53.2

WAG69C11

GCT0049

61.1

WAG43G6

WAG43N11

MCW0007

64.0

MCW0112

65.1

ADL0319

65.8

WAG67J15

LDHB

67.1

WAG53E23

WAG25G16

LEI0071

75.8

LEI0101

80.6

Chromosome 2

WAG26B13

WAG12M4

WAG21J8

WAG18L21

WAG18G1

WAG21J10

WAG14J6

WAG40C19

WAG29F23

WAG41C2

Chromosome 3

WAG23F21

WAG29L12

WAG35013

WAG54M22

WAG44P17

WAG13D11

WAG21I22

WAG40J15

WAG32A13

Chromosome 11

WAG35F15

WAG12F3

WAG52K20

Chromosome 12

WAG7E23

WAG40H2 1

Chromosome 13

WAG36M12

WAG59J23

WAG40H4
MCW0063 38.5

LEI0089 47.9

CZ567603 50.6

CZ566365 $\quad 52.1$

CZ566288 52.9

MCW0062 55.1

MCW0358 60.7

MCW0039 63.2

ADL0267 85.2

LEI0147 98.7

$\begin{array}{ll}\text { CZ568632 } & 0.4\end{array}$

MCW0261 0.8

CZ562990 2.1

MCW0141 2.5

CZ564604 $\quad 5.4$

CZ566991 5.8

$\begin{array}{ll}\mathrm{CZ} 567594 & 7.5\end{array}$

CZ564186 8.1

$\begin{array}{ll}\text { CZ562529 } & 12.5\end{array}$

LEI0143 0.5

LEI0110 $\quad 2.1$

ADL0123 4.9

$\begin{array}{ll}\text { CZ560506 } & 1.8\end{array}$

$\begin{array}{ll}\text { CZ564147 } & 3.4\end{array}$

$\begin{array}{ll}\text { CZ563151 } & 0.1\end{array}$

CZ569278 $\quad 1.1$

BH405315 13.6
Table 1 (continued)

\begin{tabular}{lll}
\hline BAC clone name & $\begin{array}{l}\text { Genetic marker } \\
\text { or GenBank } \\
\text { accession number }\end{array}$ & $\begin{array}{l}\text { Chicken } \\
\text { chromosome } \\
\text { location }^{\mathrm{a}} \text {, Mb }\end{array}$ \\
\hline $\begin{array}{ll}\text { Chromosome 14 } \\
\text { WAG32F10 }\end{array}$ & CZ562612 \\
WAG19G22 & CZ559843 & 3.7 \\
WAG42M3 & CZ567168 & 12.8 \\
Chromosome 15 & & 13.8 \\
WAG6D21 & CZ560123 & \\
WAG114F13 & CZ565238 & 0.7 \\
WAG21C7 & CZ567457 & 10.2 \\
\hline
\end{tabular}

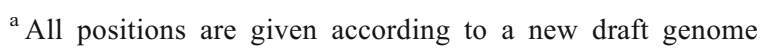
assembly (Gallus_gallus-4.0, build 3 version 1) released on 13 January 2012 (http://www.ncbi.nlm.nih.gov)

labeled with biotin-dUTP or digoxigenin-dUTP in a DOP-PCR using 6MW primer (Telenius et al. 1992). Oligonucleotide probes specific for chicken CNM (Matzke et al. 1990) and quail BglII-repeat (Tanaka et al. 2000) were also used for centromere detection, namely, CNMpos, CNMneg, and CCOneg oligonucleotides (Deryusheva et al. 2007) labeled with Cy3 or Cy5. The labeled probes were dissolved in a standard formamide hybridization buffer with an excess of salmon sperm DNA as described elsewhere (Galkina et al. 2006; Krasikova et al. 2006; Deryusheva et al. 2007).

FISH was carried out according to a DNA/(DNA+ RNA) hybridization protocol without any pretreatments of chromosomes. LBCs and probes were denatured together on the slide covered with a coverslip at $82{ }^{\circ} \mathrm{C}$ for $5 \mathrm{~min}$ followed by hybridization at $37^{\circ} \mathrm{C}$ (FISH with BAC probes) or at room temperature (FISH with oligonucleotide probes) in a humid chamber for 16-20 h. After hybridization with BACs, the slides were washed in two changes of $0.2 \times \mathrm{SSC}$ and two changes of $2 \times \mathrm{SSC}$ at $58-60{ }^{\circ} \mathrm{C}$. Avidin-Alexa 488 (Invitrogen) and antibody against digoxigenin conjugated with $\mathrm{Cy} 3$ (Jackson ImmunoResearch Laboratories) were used to detect biotin- and digoxigenin-labeled probes, respectively. After FISH with oligonucleotide probes, the slides were washed in three changes of $2 \times \mathrm{SSC}$ at $37{ }^{\circ} \mathrm{C}$. All preparations were dehydrated, air-dried, and mounted in antifade solution containing $1 \mu \mathrm{g} / \mathrm{ml} \mathrm{4',6-diamidino-2-phenyl-}$ indole (DAPI). To co-localize two or more BACs and DNA repeats on the same $\mathrm{LBC}$, we combined two-color 
FISH and re-hybridization of two sets of differentially labeled probes applied to the same slide sequentially.

\section{Microscopy}

Preparations were analyzed with a $\times 100$ objective on a Leica fluorescence microscope DM4000B equipped with a monochrome digital camera DFC350 FX and appropriate filter cubes. Fluorescent and phase contrast images were acquired with Leica CW 4000 FISH software; final figures were assembled and adjusted with Photoshop (Adobe Systems).

\section{Results}

For comparative gene mapping, we applied 41 chicken BAC probes to chicken and Japanese quail LBCs. Nearly $85 \%$ of the probes hybridized to quail LBCs in our experiments. This efficiency of heterologous hybridization corresponds to that previously observed for FISH with large-size chicken probes on metaphase chromosomes from different galliform species (Shibusawa et al. 2001, 2002; Kasai et al. 2003; Schmid et al. 2005; Kayang et al. 2006; Griffin et al. 2008). The high-resolution comparative FISH mapping combined with fine detection of centromere positions on lampbrush chromosomes allows us to determine gene order relative to centromeres most precisely. We have analyzed chromosomal rearrangements in chicken and quail by taking the three largest macrochromosomes and five microchromosomes. Because of heterochromatin accumulation, quail microchromosomes are typically much longer than corresponding chicken orthologs; the hetrochromatic arms additionally vary in length (Krasikova et al. 2009). This makes it difficult to number quail microchromosomes according to their relative size. For now, we identified and described five largest microchromosomes at the lampbrush stage in chicken and assigned chicken chromosome numbers to their quail orthologs.

\section{Chromosome 1}

Seven BAC clones (Table 1), covering the region from 53.2 to $80.6 \mathrm{Mb}$ in the current GGA1 sequence assembly (build 3.1, http://www.ncbi.nlm.nih.gov), successfully hybridized on both chicken and quail
LBCs. The FISH signals showed exactly the same order on GGA1 and CCO1 (Figs. 1a and 2). At the same time, their positions relative to centromeres are different in the two species. In chicken, five BAC clones hybridized to the short arm and two BACs hybridized to the long arm, so that the centromere on GGA1 is flanked by WAG67J15 and WAG53E23. In Japanese quail, all seven BACs hybridized to the long arm only; the centromere is located next to WAG31B10. This means that centromere regions on GGA1 and CCO1 are surrounded by different genetic material. These results suggest that the centromere repositioning was not induced by a pericentric inversion, as earlier proposed to explain the difference between two species (Shibusawa et al. 2001; Schmid et al. 2005; Kayang et al. 2006).

\section{Chromosome 2}

The existence of large-scale inversion that distinguishes chromosomes 2 in chicken and Japanese quail karyotypes has been repeatedly demonstrated by genetic and physical gene mapping (Shibusawa et al. 2001; Schmid et al. 2005; Kayang et al. 2006; Sasazaki et al. 2006). High-resolution FISH mapping on LBCs confirmed a true pericentric inversion in the case of chromosome 2 (Fig. 1b). Collectively, our data allowed us to narrow down breakpoints of the largescale pericentric inversion to the following regions in the current GGA2 sequence assembly: One corresponds to a position between $38.5 \mathrm{Mb}$ (WAG26B13) and $47.9 \mathrm{Mb}$ (WAG12M4) and the other, to a position between 85.2 Mb (WAG29F23) and 97.0 Mb (a marker in gene Spir-1 previously mapped by Sasazaki et al. 2006). Furthermore, detailed analysis of nearcentromeric regions in GGA2 and $\mathrm{CCO} 2$ showed the centromeres to be located in the same genetic context in both species (Fig. 1b); BAC WAG18L21 seems to comprise pericentromeric sequences. Intriguingly, the pericentric BAC, WAG18L21, and two adjoined ones (WAG21J8 and WAG12M4) showed identical hybridization pattern in chicken and Japanese quail, as if this small region within the large-scale pericentric inversion was inverted again.

\section{Chromosome 3}

Comparison of linkage maps previously constructed for chicken and quail genomes has revealed a potentially 
Fig. 1 Comparative mapping of chicken BAC clones to chicken (GGA) and Japanese quail (CCO) lampbrush chromosomes 1 (a) and 2 (b). Positions of BAC clones are assigned to cytological maps of chromomeric pattern of corresponding lampbrush chromosomes. DAPIstained prominent chromomeres are indicated as black axial dots. The red circles indicate the centromeric cohesin-enriched granules (CEN). To stress the homology between GGA2p and the distal part of $\mathrm{CCO} 2 \mathrm{q}$ as well as the distal part of GGA2q and CCO2p, quail LBC2 map is inverted. The dashed lines indicate the boundaries of a large-scale pericentric inversion between GGA2 and $\mathrm{CCO} 2$. $\mathrm{BAC}$ clones within the large inverted region that have identical order in both species are depicted with magenta. BAC clones hybridized only to chicken LBCs are depicted with grey. Cytological landmarks: TBL telomere bow-like loops, PBL11 marker loop on chicken LBC1, SM spaghetti marker, $L L$ lumpy loops

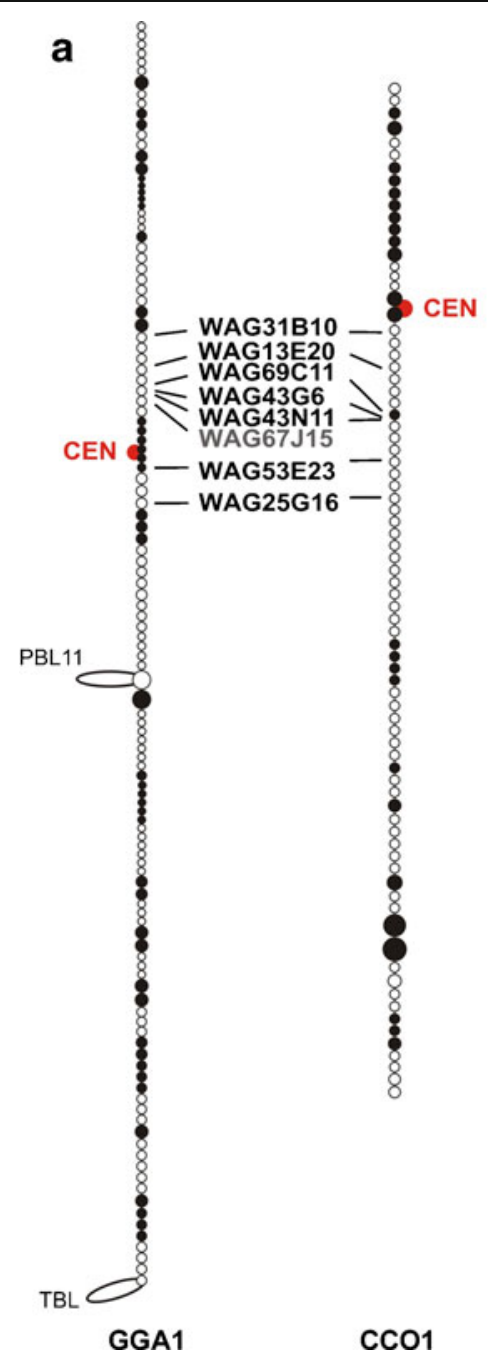

inverted region on chromosome 3 (Sasazaki et al. 2006). Comparative FISH mapping of nine BAC clones on chicken and quail LBCs allowed us to verify this inversion and to define its breakpoints precisely (Fig. 3). BAC clones WAG13D11, WAG21I22, WAG40J15, and WAG32A13 had an identical order in GGA3 and CCO3, while WAG23F21, WAG29L12, WAG35O13, WAG54M22, and WAG44P17 showed an inverted order in $\mathrm{CCO} 3$ as compared with GGA3 (Fig. 3c). WAG44P17 and WAG13D11 hybridized very close together on GGA3 but were separated by six chromomeres on $\mathrm{CCO} 3$ with WAG44P17 located terminally (Fig. 3). In chicken, WAG23F21 is a terminally hybridized probe on $\mathrm{LBC} 3$. Thus, the breakpoint of the inversion appears to localize in a very narrow region at position $5.4-5.8 \mathrm{Mb}$. It is worth noting that, in the current GGA3 sequence assembly, a gap exists at

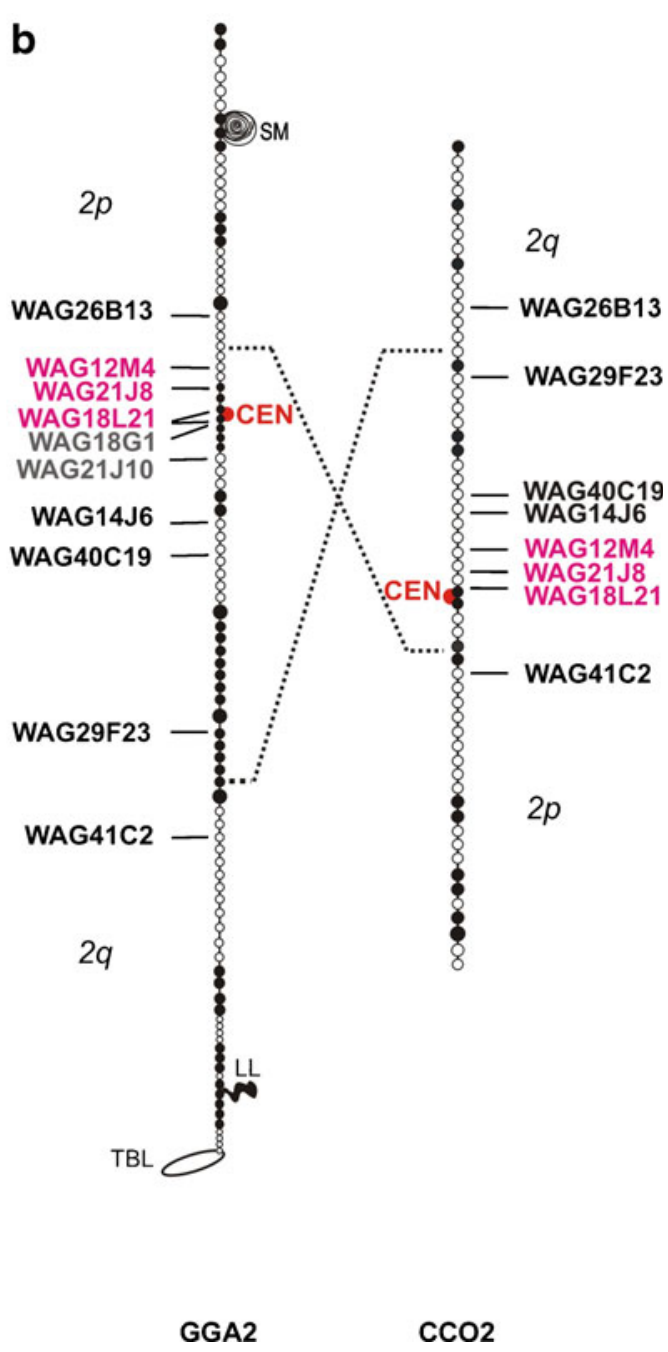

position 5.6 Mb. This gap was previously shown to contain a CNM repeat cluster (Zlotina et al. 2010).

When we determined the inversion of centromere bearing region in chromosome 3 , we decided to look closely at the sequences surrounding centromeres in GGA3 and CCO3. In chicken, the centromere position marked with a cohesin-enriched granule on LBC3 was assigned to position $2.4 \mathrm{Mb}$ in the GGA3 sequence assembly (Zlotina et al. 2010). This position was shown to associate with WAG35O13- and WAG54M22-positive chromomeres. In the case of quail chromosome 3, centromere is located at the most terminal prominent chromomere (Krasikova et al. 2006, Fig. 3); the other flank centromere-adjacent chromomere hybridizes with BAC WAG44P17 whereas WAG35O13- and WAG54M22positive region is two chromomeres away (Fig. 3). Thus, different genetic markers flank centromeres on 
Fig. 2 High-resolution twocolor FISH on chicken $(\mathbf{a}, \mathbf{b})$ and Japanese quail LBC1 $\left(\mathbf{a}^{\prime}, \mathbf{b}^{\prime}\right)$. FISH signals for chicken BAC clones WAG31B10 (red),

WAG43G6 (green),

WAG53E23 (green), and

WAG25G16 (red) are shown on top of LBC phase contrast images and pointed out with white arrows.

White arrowheads indicate centromere positions. Scale bars $=10 \mu \mathrm{m}$
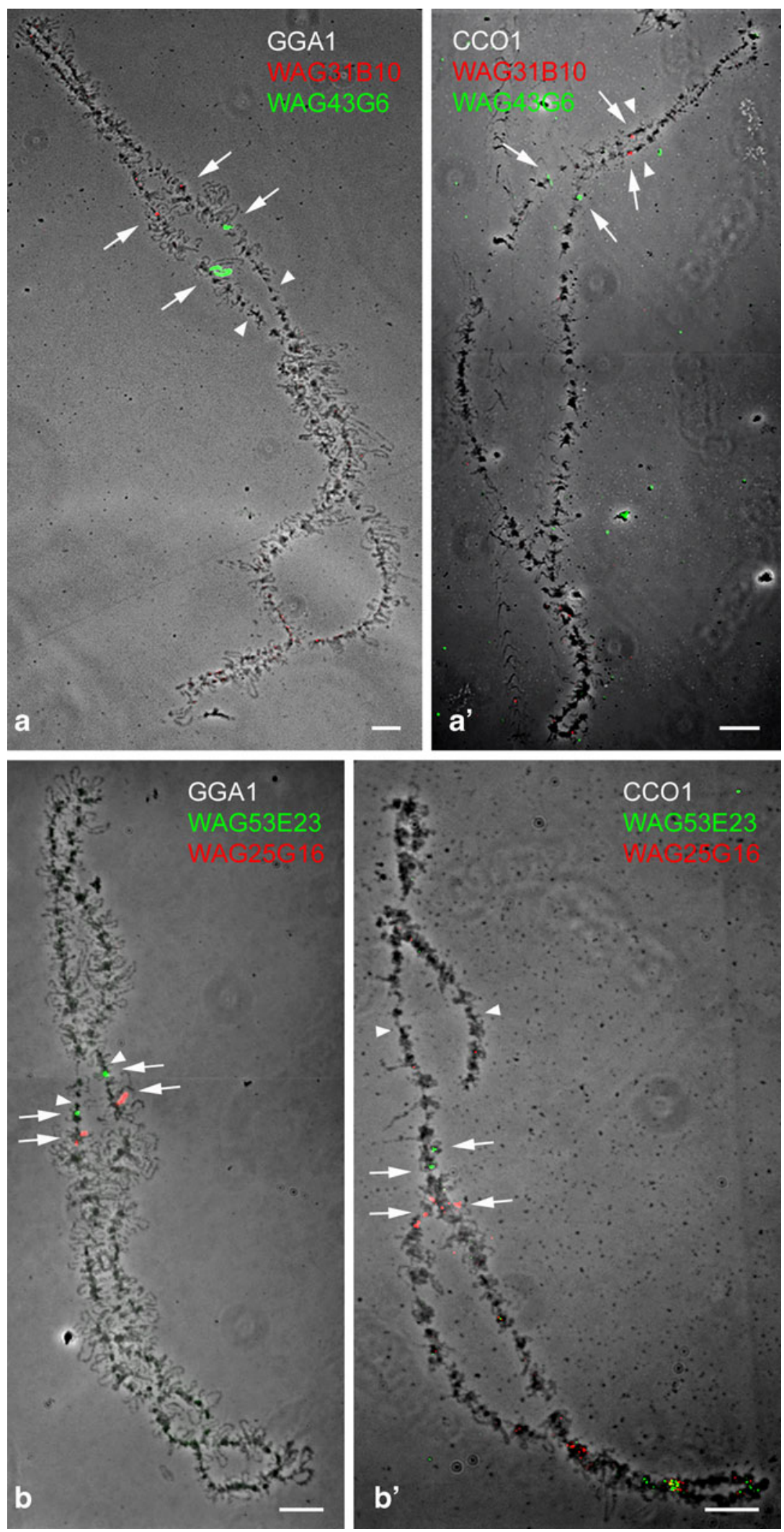
Fig. 3 High-resolution FISH mapping on chicken and Japanese quail LBC3. (a, b) Two-color FISH with BAC clones WAG44P17 (green) and WAG13D11 (red) to chicken (a) and quail LBC3 (b). Chromosomes are counterstained with DAPI (blue). Arrowheads indicate centromere positions. Scale bars $=$ $10 \mu \mathrm{m}$. c Localization of chicken BAC clones and CNM-repeat clusters on cytological maps of chicken and quail LBC3. All

indications are the same as in Fig. 1. Dashed lines depict the inversion between GGA3 and CCO3
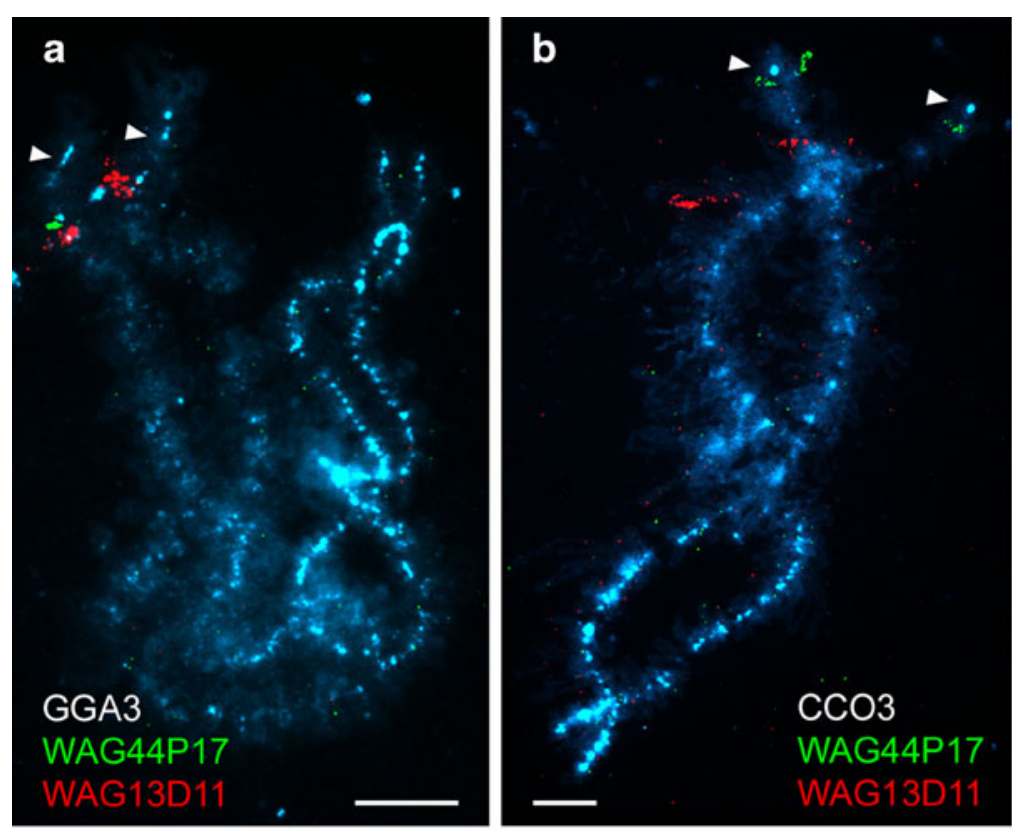

c

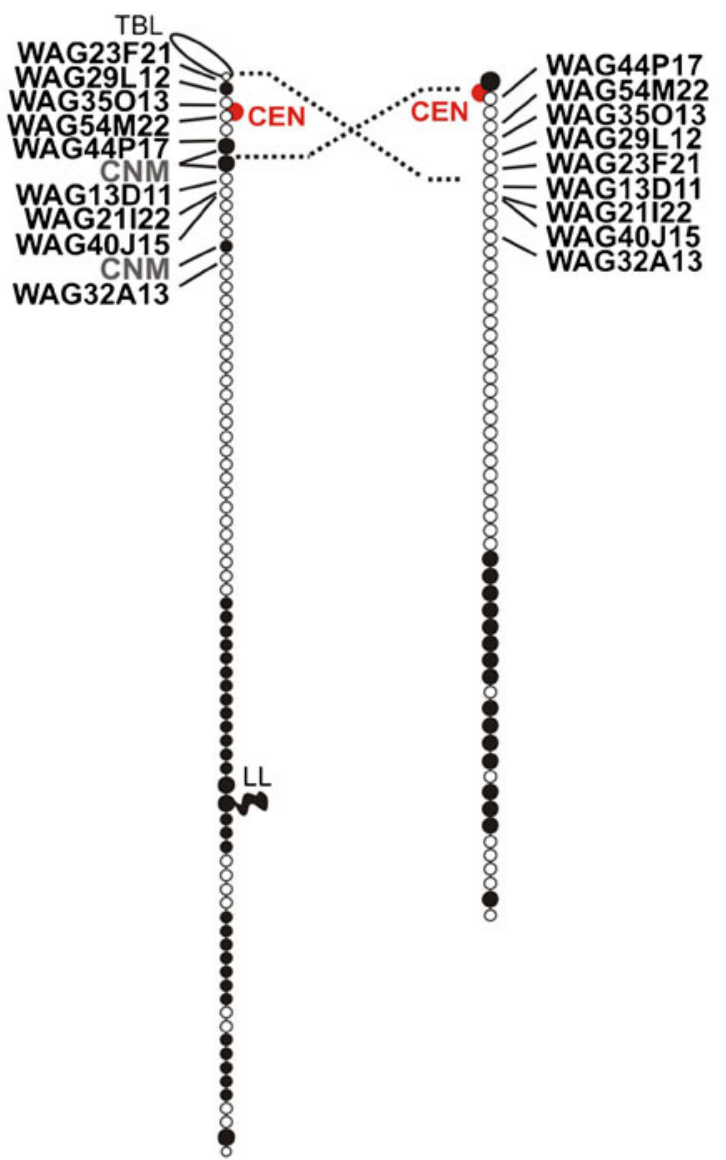

GGA3

$\mathrm{CCO} 3$ 
GGA3 and CCO3. We suggest that the centromere repositioning to a different genetic environment cannot be explained by the inversion we detected.

\section{Microchromosomes}

It is worth noting that, except for chicken NORbearing chromosome 16 (Solinhac et al. 2010), none of chicken and quail microchromosomes at the lampbrush stage have been identified so far and studied in detail. The first essential step in this comparative mapping study was identification and morphological description of corresponding LBCs.
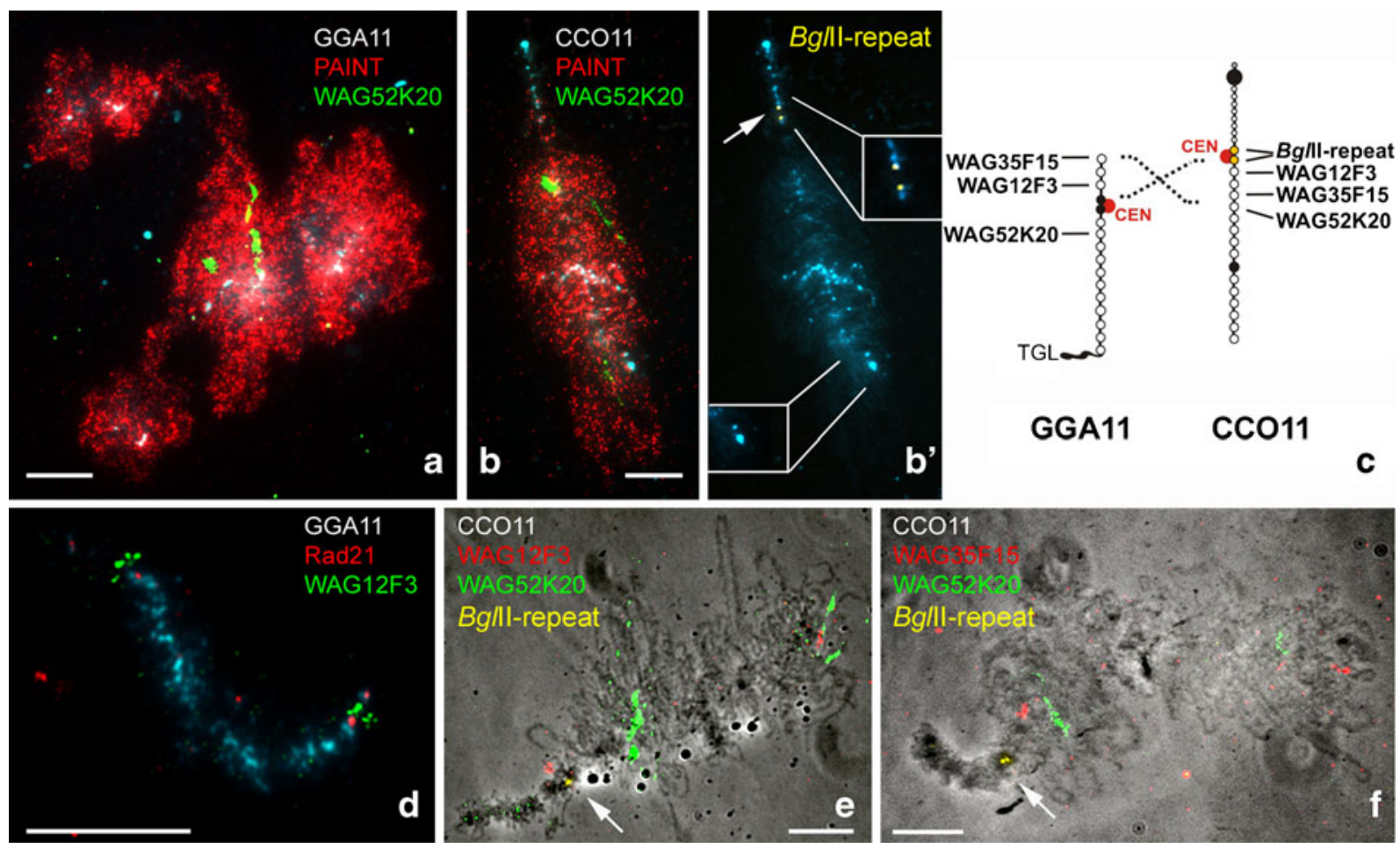

Fig. 4 Comparative cytogenetic analysis of chicken and Japanese quail chromosome 11 at the lampbrush stage. a, b Identification of chicken (a) and quail (b) LBC11 using FISH with chicken whole chromosome paint F12 (red) and BAC clone WAG52K20 (green). (b') Re-FISH with a BglII-repeat probe (yellow) on the same quail chromosome shown in $\mathbf{b}$. This quail chromosome represents asymmetric bivalent 11 with polymorphic heterochromatic short arms. One of the homologs is submetacentric with BgIII-repeat cluster (arrow) in the centromeric region (insert on the right); the other homolog is acrocen-

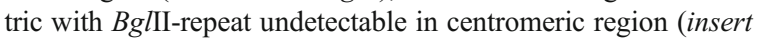
on the left). $\mathbf{c}, \mathbf{d}, \mathbf{e}, \mathbf{f}$ Centromere positioning on chicken and quail LBC11 relative to chicken molecular markers. c Cytological maps of chicken and quail LBC11 with chicken BAC clones
BAC clones specific for GGA11 hybridized to a single relatively large microbivalent (Fig. 4). Surprisingly, in chicken, it was the same LBC that was previously characterized as chromosome 10 (Krasikova et al. 2006) using painting probe F12 (Griffin et al. 1999). This chromosome has unique features: It is the only submetacentric microchromosome in chicken that additionally lacks microchromosomal centromere-specific CNMrepeat (Krasikova et al. 2006). To verify whether we are dealing with the same chromosome, we applied F12 paint and GGA11-specific BAC probes to LBCs simultaneously. They hybridized together on the same LBC (Fig. 4a). We assume F12 painting probe was misannotated because of difficulties with microchromosome 
identification. When we identified GGA11 and its quail ortholog (Fig. 4b), we constructed cytological maps for chicken and quail LBC11 (Fig. 4c). Though chicken and quail LBC11 are poor with landmarks, these working maps are useful for further high-resolution comparative gene mapping.

Here, we compared centromere positions relative to molecular markers in chicken and quail LBC11 (Fig. 4c, $\mathrm{d}, \mathrm{e}, \mathrm{f}$ ); centromeres were mapped by immunostaining for centromeric cohesin-enriched granules and using FISH with a quail centromeric BglII-repeat probe. Though chromosome 11 is submetacentric both in chicken and quail, the nature of the short arm seems to be different in the two species. The short arm of GGA11 appears to be euchromatic: BACs WAG35F15 and WAG12F3 hybridized to the short arm. The short arm of CCO11 is formed by partially decondensed heterochromatin previously described to be typical of quail microchromosomes (Krasikova et al. 2009); all GGA11-specific BACs we used hybridized to the long arm of CCO11 (Fig. 4c, e, f). In the previous studies of quail LBCs (Deryusheva et al. 2007; Krasikova et al. 2009), we observed polymorphism in the length of the short arms of certain microchromosomes. In some extreme cases, one homolog in a bivalent was metacentric while the other, acrocentric with centromeric $B g / I I-$ repeat being lost along with heterochromatic short arm. Now, we can identify this atypical chromosome pair as CCO11 (Fig. 4b').

The hybridization pattern of three BACs mapped on GGA11 and CCO11 suggests an inversion that involves the genetic material of the short arm of GGA11 (Fig. 4). Interestingly, a similar inversion was revealed between GGA11 and its turkey ortholog MGA13 (Dalloul et al. 2010; Zhang et al. 2011).

The next LBC we identified and analyzed in chicken and Japanese quail was chromosome 13, for which a well-characterized whole-chromosome paint (Masabanda et al. 2004) and BAC clones were available. Cytological maps of chicken and quail LBC13 with assigned BACs are shown in Fig. 5. In the current GGA13 sequence assembly, centromere position relative to molecular markers is ambiguous. Chicken LBC13 represents a typical acrocentric chromosome with a centromeric cohesin-enriched granule detectable near prominent terminal DAPI-positive chromomeres comprising a CNM repeat cluster. BAC clone WAG59J23 (1.1 Mb) hybridized very close to the centromeric granule (Fig. 5d) and the CNM-repeat cluster (Fig. 5e). Contrarily, on quail LBC13,
WAG59J23 was mapped to the most terminal chromomere on the long arm; centromere position was identified by FISH with a $B g /$ III-repeat probe specific for quail centromere (Fig. 5f). BAC clone WAG40H4 $(13.6 \mathrm{Mb})$ hybridized as expected to the terminal region of chicken LBC13 whereas, in quail, this probe hybridized to the proximal region of the long arm, only few chromomeres away from centromere (Fig. 5g). Thus, the mapped BAC clones show an inverse order relative to centromere in chicken and quail chromosome 13; centromeres in GGA13 and CCO13 appear to be flanked by different genetic markers.

Similarly, two BAC clones from near-centromeric region of chicken chromosome 12 hybridized to quail LBC12 in an inverted order at the distal region of the long arm (Fig. 5a, b, c). This suggests that centromeres of GGA12 and CCO12 are in a different genetic context as in the case of chromosome 13.

Chromosome 14 is another example of microchromosomes whose morphology is different in chicken and quail. Working cytological maps of chicken and quail LBCs 14 are shown in Fig. 6c. Three BAC clones were mapped to chicken LBC14. Surprisingly, BAC clones WAG19G22 and WAG42M3, assigned to positions 12.8 and $13.8 \mathrm{Mb}$, respectively, in the current GGA14 sequence assembly, hybridized next to a CNM-positive chromomere, which corresponds to the position of centromere (Fig. 6a, c). BAC WAG32F10 $(3.7 \mathrm{Mb})$ from the other end of GGA14 linkage group hybridized to the distal region of LBC14 (Fig. 6a, c). This hybridization pattern suggests that the actual gene order relative to centromere position in GGA14 is opposite to the annotation in the current chicken genome sequence assembly.

FISH with two BACs WAG19G22 and WAG32F10 to LBCs allowed us to identify quail ortholog of GGA14. These BACs hybridized to the long arm of CCO14 but in reverse order, as compare with GGA14 (Fig. 6b, c). Despite the detected intrachromosomal rearrangement, the difference in centromeric index between GGA14 and CCO14 is the result of heterochromatic short arm formation in quail.

The last chromosome we analyzed was microchromosome 15. Three markers from GGA15 were mapped to chicken LBC15. Similarly to GGA14, BAC WAG21C7 assigned to position $11.8 \mathrm{Mb}$ in the current GGA15 sequence assembly hybridized to lateral loops at the CNM-positive centromeric 

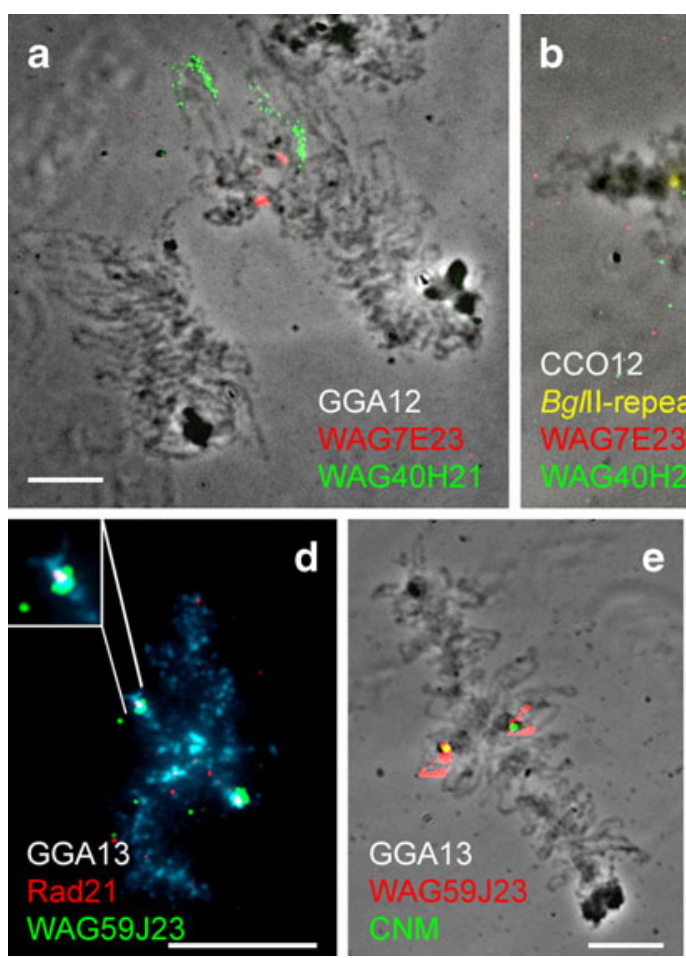

e

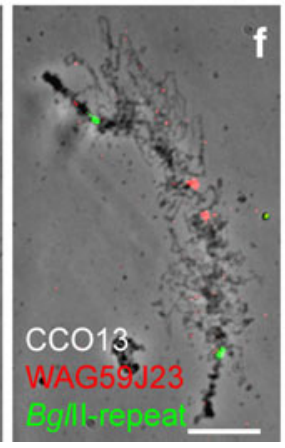

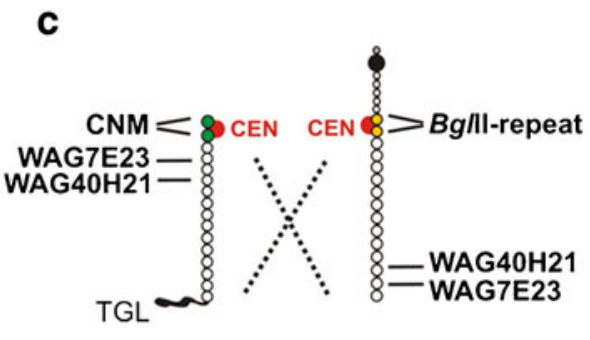

GGA12

\section{$\operatorname{Cc012}$}

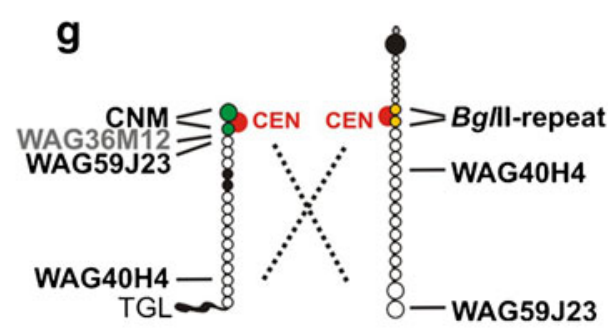

GGA13

\section{$\operatorname{cc013}$}

Fig. 5 Comparative cytogenetic analysis of chicken and Japanese quail chromosomes 12 and 13 at the lampbrush stage. a, b, c Comparative FISH mapping of chicken BAC clones WAG7E23 (red) and WAG40H21 (green) to chicken (a) and quail (b) LBC12. Centromere positions on quail LBC12 are detected by FISH with a BglII-repeat probe (yellow). c Localization of the mapped BAC clones is depicted on GGA12 and CCO12 cytological maps. d, e, f, g Comparative FISH mapping of chicken BAC clones to chicken and quail LBC13. d FISH with BAC clone WAG59J23 (green) to chicken LBC13 after immunofluorescent detection of a centromeric granule (red) using antibodies against $\operatorname{Rad} 21$. Insert shows enlarged centromeric region with fluorescent

chromomere (Fig. 6d), while BAC WAG6D21 $(0.7 \mathrm{Mb})$ hybridized to the $\mathrm{q}$ terminus of LBC15 (Fig. 6f). Quite predictably, BAC WAG114F13 $(10.2 \mathrm{Mb})$ hybridized to the proximal region of LBC15, between WAG6D21 and WAG21C7, in the close proximity to the latter one. Thus, similar to GGA14, gene order in GGA15 relative to centromere position is misannotated in the current chicken genome sequence assembly. Centromere positions determined on radiation hybrid maps of GGA14 and GGA15 (Alain Vignal, personal communication) also conform to our findings. Therefore, we suggest that gene order in the current GGA14 and GGA15 sequence assembly signals. e, f FISH with BAC clone WAG59J23 (red) to chicken (e) and quail (f) LBC13 followed by FISH mapping of centromere-specific CNM (green) and BglII-repeat (green), respectively. g Localization of the mapped BAC clones is depicted on GGA13 and CCO13 cytological maps. $\mathrm{CNM}$ and BglII-repeat-bearing chromomeres are shown in green and yellow, respectively. Dashed lines indicate the inverted gene order in Japanese quail chromosomes as compared with chicken chromosomes. The rest of indications are the same as in Fig. 1. Chromosomes are counterstained with DAPI (d) or fluorescent signals are shown on the corresponding phase contrast images (a, b, e, f). Scale bars $=10 \mu \mathrm{m}$

should be flipped to make it consistent with other annotated chromosomes.

Only one out of three GGA15-specific BACs hybridized successfully to quail LBCs. This allowed us to identify quail ortholog of GGA15. Similar to FISH on GGA15, BAC WAG21C7 hybridized to the long arm of CCO15 close to centromere position, namely, at the chromomere adjacent to a centromeric $B g / I I-$ repeat cluster (Fig. 6e, f). Thus, we assume that centromeres in chicken and quail chromosome 15 co-localize with similar genetic markers, and the difference in chromosome morphology between these two species is trigged by the formation of heterochromatic short arms, which is typical of quail microchromosomes. 

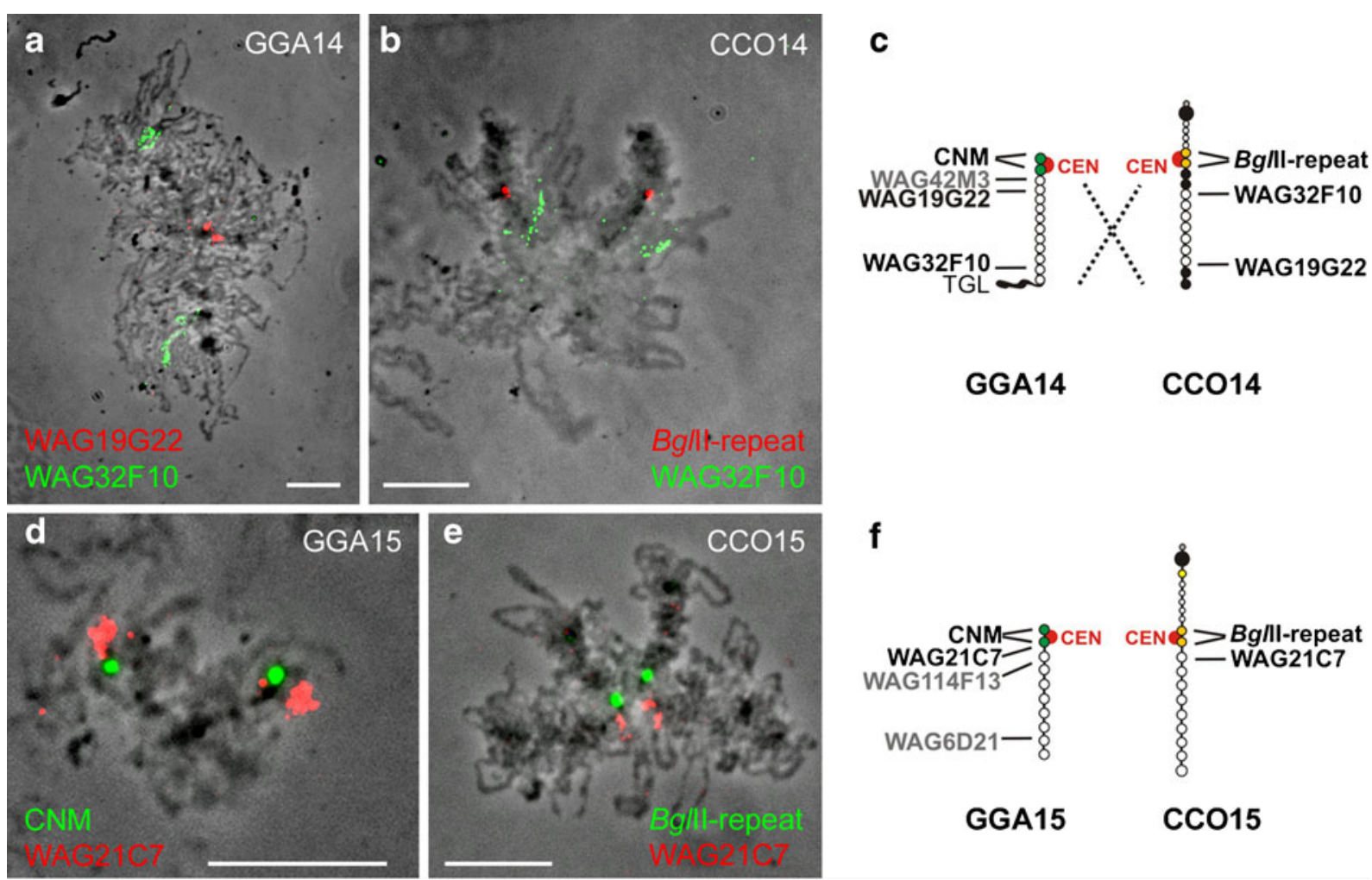

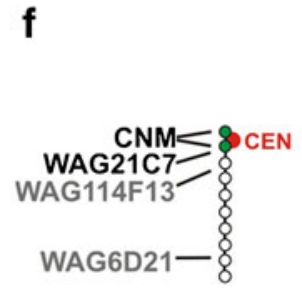

GGA15

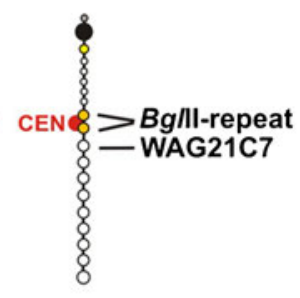

CC015
Fig. 6 Comparative cytogenetic analysis of chicken and Japanese quail chromosomes $14(\mathbf{a}, \mathbf{b}, \mathbf{c})$ and $15(\mathbf{d}, \mathbf{e}, \mathbf{f})$ at the lampbrush stage. a FISH with chicken BAC clones WAG19G22 (red) and WAG32F10 (green) to chicken LBC14. b FISH with BAC WAG32F10 (green) and a BglII-repeat probe (red) to quail LBC14. c Localization of the mapped BAC clones is depicted on GGA14 and CCO14 cytological maps. d, e Comparative

\section{Discussion}

Very high conservation of synteny demonstrated for the chicken and Japanese quail (Shibusawa et al. 2001; Guttenbach et al. 2003; Schmid et al. 2005; Kayang et al. 2006; Sasazaki et al. 2006) is quite typical of bird karyotypes (Derjusheva et al. 2004; Schmid et al. 2005; Griffin et al. 2007; Nanda et al. 2008). At the same time, the rate of intrachromosomal rearrangements that change gene order seems not to be low. We verified and extended previous data on chromosomal rearrangements between chicken and Japanese quail using high-resolution comparative FISH on LBCs. The main conclusion emerging from our observations is that pericentric inversions are not the major mechanism of chromosome morphology alteration in chicken and quail karyotypes. In fact, microchromosomes in quail must have become biarmed through
FISH mapping of BAC WAG21C7 (red) to chicken (d) and quail (e) LBC15 followed by FISH with CNM (green) or BglII-repeat (green) probes, respectively. f Localization of the mapped BAC clones is depicted on GGA15 and CCO15 cytological maps. All indications are the same as in Figs. 1 and 5. Hybridization signals are shown on the corresponding phase contrast images. Scale bars $=10 \mu \mathrm{m}$

heterochromatin accumulation, which forms entire short arms, as compared with corresponding chicken acrocentric microchromosomes (Krasikova et al. 2009, this study). Centromere repositioning in chromosomes 1 (Fig. 1) and 4 (Galkina et al. 2006) has not been accompanied by gene reshuffling; centromeres of these chromosomes in chicken and Japanese quail appear to have formed de novo. Another example is chromosome 3 rearrangement. Although the chromosomal rearrangement between GGA3 and $\mathrm{CCO} 3$ we identified should be considered as a pericentric inversion, an independent event, namely centromere repositioning to a different genetic location, must have driven the change of chromosome morphology. De novo centromere formation has been earlier proposed for chromosome 4 in red-legged partridge (Kasai et al. 2003) and chromosome $\mathrm{Z}$ in Pekin duck as compare with chicken orthologs (Skinner et al. 2009). 
The centromere repositioning during karyotype evolution, the so-called evolutionary new centromere (ENC) formation, is rather a common phenomenon. It has been repeatedly described in different taxonomic groups as diverged as mammals, birds, and plants (Kasai et al. 2003; Nagaki et al. 2004; O'Neill et al. 2004; Galkina et al. 2006; Han et al. 2009; Skinner et al. 2009; Rocchi et al. 2012). Though mechanisms of ENC formation are obscure, an attractive hypothesis implies the following events: old centromere inactivation and neocentromere formation in a new euchromatic locus, with fixation of these first steps in population. The next events are the disappearance of satellite DNA at the old inactivated centromeres and accumulation of new satellite DNA at the new centromere positions with an evident elimination of their neocentromeric nature (Amor et al. 2004; Marshall et al. 2008; Piras et al. 2010). Does centromere repositioning in birds fit this model? Can we trace steps of ENC formation?

For a long time, the information about centromerespecific sequences in birds was limited to a few satellites identified in several species (Matzke et al. 1990; Solovei et al. 1996; Tanaka et al. 2000; Saifitdinova et al. 2001; Wang et al. 2002; Krasikova et al. 2006; Deryusheva et al. 2007). Even in extensively studied galliform species, such as chicken and quail, primarily microchromosome-specific centromeric repeats were well characterized (Matzke et al. 1990; Tanaka et al. 2000; Krasikova et al. 2006; Deryusheva et al. 2007). A recent comprehensive analysis revealed elusive centromere DNA specific for chicken chromosomes (Shang et al. 2010). We expected to see a difference in centromere organization between chromosomes with centromere position preserved in the same genetic environment during karyotype evolution (chromosome 2) and chromosomes with centromeres shifted along chromosomes (chromosomes 1, 3, and 4). Surprisingly, these chromosomes have similar genome organization of their centromere regions: Centromeric sequences are organized in typical tandem repeat arrays and must have derived from a common ancestor, at least partially (Shang et al. 2010). Given the fact that more than 30 million years separate the chicken and Japanese quail, chicken centromeres could accumulate satellite repeats being transformed into "mature centromeres." On the other hand, chicken centromeres might represent ancestral centromeres whereas ENC formation occurred in quail karyotype evolution. Without extensive comparative analysis including centromere positioning on orthologous chromosomes from outgroup karyotypes, the directionality of ENC formation is ambiguous. Sex chromosome $\mathrm{Z}$ is the most frequently and extensively rearranged chromosome in bird karyotypes (Griffin et al. 2007; Nanda et al. 2008). Centromere repositioning was predicted for chromosome $\mathrm{Z}$ when Pekin duck and chicken karyotypes were compared (Skinner et al. 2009). In fact, non-tandem-repeat sequences were found to form functional centromere of chicken chromosome Z (Shang et al. 2010). This type of centromere organization is expected for early events of centromere formation. Another candidate for finding of newly formed centromere is chromosome 5. The centromere of this chromosome in chicken also does not contain tandem repeats typical of well-established centromeres (Shang et al. 2010).

Microchromosomal counterparts of chicken and quail karyotypes are designated by conserved centromeric repeats CNM and BglII-repeat, respectively, which must have originated from a common ancestral sequence. Does it indicate that centromere repositioning is characteristic of macrochromosomes only? The pattern of comparative FISH mapping on chicken and quail LBCs 12, 13, and 14 (Figs. 5 and 6) poses a question whether these chromosomes are examples of centromere repositioning events in microchromosomes or examples of large-scale paracentric inversions. Intriguingly, a subset of chicken microchromosomes show the presence of CNM repeat at both termini: centromeric and q arm-terminal clusters (Krasikova et al. 2006; Deryusheva et al. 2007). Three possibilities could be hypothesized based on this observation: (1) CNM repeat marked originally both chromosomal ends that allowed stochastic fixation of centromere position, (2) recent centromere repositioning occurred via CNM duplication, and the telomeric CNM cluster represents a residual array at the old inactivated centromere, (3) paracentric inversions spanning the whole arm with breakpoints in the CNM cluster occurred in chicken karyotype evolution.

Molecular mechanisms underlying chromosomal rearrangements in birds are poorly understood. Although little is known about the sequences from breakpoint regions, the speculations that repetitive DNA is involved in genome reshuffling in birds sound reasonable. In fact, satellite DNA is believed to have promoted chromosomal rearrangements in mammals 
(Slamovits and Rossi 2002; Ruiz-Herrera et al. 2006; Adega et al. 2009). In birds, the breakpoints of the inversion, which distinguish GGA3 and $\mathrm{CCO} 3$, were mapped to positions occupied by long clusters of tandem repeats: $\mathrm{CNM}$ repeat at position $5.6 \mathrm{Mb}$ in the current GGA3 sequence assembly (Fig. 3, Zlotina et al. 2010) and $Z$ chromosome-specific macrosatellite at telomere bow-like loops (TBL) (Hori et al. 1996). Strikingly, the position 5.6 Mb in GGA3 was identified as a breakpoint of an inversion between GGA3 and its turkey ortholog MGA2 with the other breakpoint located in GGA3 at position 11.6 Mb (Dalloul et al. 2010; Zhang et al. 2011), which is another CNMenriched region (Zlotina et al. 2010). The second inversion between GGA3 and MGA2 was predicted to have a breakpoint at position $2.4 \mathrm{Mb}$ in GGA3 (Dalloul et al. 2010; Zhang et al. 2011). This position corresponds to the centromere of GGA3 (Zlotina et al. 2010) and also comprises of satellite repeats (Shang et al. 2010). Numerous repetitive sequences of different classes have been revealed in evolutionary breakpoints in chicken, turkey, and zebra finch (Itoh et al. 2011; Skinner and Griffin 2011). One third of the identified breakpoint regions are thought to have been "reused" during avian genome evolution. These results are consistent with a widespread "fragile-breakage" model of chromosomal evolution implying a nonrandom breakpoint distribution and the existence of chromosomal regions prone to breakage in mammalian genomes (Pevzner and Tesler 2003; Murphy et al. 2005; Kemkemer et al. 2009). However, what is a primary event in chromosomal rearrangements: Accumulation of repeated sequences produces a fragile site, or repetitive sequences accumulate at sites of chromosome breaks remains an open question.

A strong association between structural genomic variations and increased recombination rates has been recently revealed; non-allelic homologous recombination is proposed to play a role in chromosomal rearrangements in avian genome evolution (Völker et al. 2010; Skinner and Griffin 2011). Indeed, CNM clusters on GGA3 that are associated with chromosomal breakpoints may be considered as recombination hot spots. A high-density SNP-based linkage map of the chicken genome revealed elevated recombination rates in these chromosomal regions (Groenen et al. 2009). Analysis of chiasma distribution along chicken LBC3, which also allows us to estimate frequency of recombination, supports this assumption (Figure S1). We do not exclude that recombination activity could promote chromosomal reshuffling. On the other hand, high frequency of recombination may be a consequence of tandem repeat accumulation at the breakpoints. Similarly, a recombination hot spot was identified in chicken LBC16 at position of the long arrays of PO41 tandem repeat (Solinhac et al. 2010). Despite intensive studies of the genomic organization in a wide variety of different species, with many genomes fully sequenced, the main trigger(s) of evolutionary genome rearrangements remains obscure.

Based on our findings and earlier data, we suggest that intrachromosomal rearrangements that accompanied avian karyotype evolution are more diverse than previously thought. In conclusion, we demonstrated again that giant lampbrush chromosomes represent a reliable system for high-resolution physical gene mapping and the detail comparative cytogenetic analysis of avian karyotypes.

Acknowledgments We are grateful to Dr. F. Habermann (Ludwig-Maximilians-University of Munich, Germany) and Prof. D. Griffin (School of Biosciences, University of Kent, Canterbury, UK) for providing chicken chromosome-specific paints. We especially thank Herbert Macgregor for careful editing of the manuscript. The equipment we used was provided by Core Facility "Chromas" (Saint-Petersburg State University). This work was supported by the Russian Foundation for Basic Research (grants 08-04-00493a and 11-04-01347a) and the Russian President's Program for Support of Leading Scientific Schools (project \#4385.2012.4).

\section{References}

Adega F, Guedes-Pinto H, Chaves R (2009) Satellite DNA in the karyotype evolution of domestic animals - clinical considerations. Cytogenet Genome Res 126:12-20

Amor DJ, Bentley K, Ryan J et al (2004) Human centromere repositioning "in progress". Proc Natl Acad Sci USA 101:6542-6547

Calderón PL, Pigozzi MI (2006) MLH1-focus mapping in birds shows equal recombination between sexes and diversity of crossover patterns. Chromosome Res 14:605-612

Callan HG (1986) Lampbrush chromosomes. Mol Biol Biochem Biophys 36:1-254

Crooijmans RPMA, Vrebalov J, Dijkhof RJ et al (2000) Twodimensional screening of the Wageningen chicken BAC library. Mamm Genome 11:360-363

Dalloul RA, Long JA, Zimin AV et al (2010) Multi-platform next-generation sequencing of the domestic turkey (Meleagris gallopavo): genome assembly and analysis. PLoS Biol 8:e1000475

Derjusheva S, Kurganova A, Habermann F, Gaginskaya E (2004) High chromosome conservation detected by 
comparative chromosome painting in chicken, pigeon and passerine birds. Chromosome Res 12:715-723

Deryusheva S, Krasikova A, Kulikova T, Gaginskaya E (2007) Tandem 41-bp repeats in chicken and Japanese quail genomes: FISH mapping and transcription analysis on lampbrush chromosomes. Chromosoma 116:519-530

Gaginskaya E, Kulikova T, Krasikova A (2009) Avian lampbrush chromosomes: a powerful tool for exploration of genome expression. Cytogenet Genome Res 124:251-267

Galkina S, Deryusheva S, Fillon V et al (2006) FISH on avian lampbrush chromosomes produces higher resolution gene mapping. Genetica 128:241-251

Griffin DK, Haberman F, Masabanda J et al (1999) Micro- and macrochromosome paints generated by flow cytometry and microdissection: tools for mapping the chicken genome. Cytogenet Cell Genet 87:278-281

Griffin DK, Robertson LBW, Tempest HG, Skinner BM (2007) The evolution of the avian genome as revealed by comparative molecular cytogenetics. Cytogenet Genome Res 117:64-77

Griffin DK, Robertson LB, Tempest HG et al (2008) Whole genome comparative studies between chicken and turkey and their implications for avian genome evolution. BMC Genomics 9:168

Groenen MAM, Wahlberg P, Foglio M et al (2009) A highdensity SNP-based linkage map of the chicken genome reveals sequence features correlated with recombination rate. Genome Res 19:510-519

Guttenbach M, Nanda I, Feichtinger W, Masabanda JS, Griffin DK, Schmid M (2003) Comparative chromosome painting of chicken autosomal paints 1-9 in nine different bird species. Cytogenet Genome Res 103:173-184

Han Y, Zhang Z, Liu C et al (2009) Centromere repositioning in cucurbit species: implication of the genomic impact from centromere activation and inactivation. Proc Natl Acad Sci USA 106(35):14937-14941

Hori T, Suzuki Y, Solovei I et al (1996) Characterization of DNA sequence constituting the terminal heterochromatin of the chicken $\mathrm{Z}$ chromosome. Chromosome Res 4:411426

Itoh Y, Kampf K, Arnold AP (2011) Possible differences in the two $\mathrm{Z}$ chromosomes in male chickens and evolution of MHM sequences in Galliformes. Chromosoma 120:587598

Kaelbling M, Fechheimer NS (1983) Synaptonemal complexes and the chromosomes of the domestic fowl, Gallus domesticus. Cytogenet Cell Genet 35:87-92

Kasai F, Garcia C, Arruga MV, Ferguson-Smith MA (2003) Chromosome homology between chicken (Gallus gallus domesticus) and the red-legged partridge (Alectoris rufa); evidence of the occurrence of a neocentromere during evolution. Cytogenet Genome Res 102:326-330

Kayang BB, Fillon V, Inoue-Murayama M et al (2006) Integrated maps in quail (Coturnix japonica) confirm the high degree of synteny conservation with chicken (Gallus gallus) despite 35 million years of divergence. BMC Genomics 7:101

Kemkemer C, Kohn M, Cooper DN et al (2009) Gene synteny comparisons between different vertebrates provide new insights into breakage and fusion events during mammalian karyotype evolution. MC Evol Biol 9:84
Krasikova A, Barbero JL, Gaginskaya E (2005) Cohesion proteins are present in centromere protein bodies associated with avian lampbrush chromosomes. Chromosome Res 13:675-685

Krasikova A, Deryusheva S, Galkina S, Kurganova A, Evteev A, Gaginskaya E (2006) On the positions of centromeres in chicken lampbrush chromosomes. Chromosome Res 14:777-789

Krasikova A, Daks A, Zlotina A, Gaginskaya E (2009) Polymorphic heterochromatic segments in Japanese quail microchromosomes. Cytogenet Genome Res 126:148-155

Krasikova A, Fukagawa T, Zlotina A (2012) High-resolution mapping and transcriptional activity analysis of chicken CENP-A-associated DNA sequences on giant lampbrush chromosomes. Chromosome Res (this issue)

Marshall OJ, Chueh AC, Wong LH, Choo KHA (2008) Neocentromeres: new insights into centromere structure, disease development, and karyotype evolution. Am J Hum Genet 82:261-282

Masabanda JS, Burt DW, O'Brien PCM et al (2004) Molecular cytogenetic definition of the chicken genome: the first complete avian karyotype. Genetics 166:1367-1373

Matzke MA, Virga F, Berger H et al (1990) A 41-42 bp tandemly repeated sequence isolated from nuclear envelopes of chicken erythrocytes is located predominantly on microchromosomes. Chromosoma 99:131-137

Morgan GT (2002) Lampbrush chromosomes and associated bodies: new insights into principles of nuclear structure and function. Chromosome Res 10:177-200

Murphy WJ, Larkin DM, van der Wind AE et al (2005) Dynamics of mammalian chromosome evolution inferred from multispecies comparative maps. Science 309:613617

Nagaki K, Cheng Z, Ouyang S et al (2004) Sequencing of a rice centromere uncovers active genes. Nat Genet 36:138-145

Nanda I, Schlegelmilch K, Haaf T, Schartl M, Schmid M (2008) Synteny conservation of the $\mathrm{Z}$ chromosome in 14 avian species (11 families) supports a role for $\mathrm{Z}$ dosage in aviansex determination. Cytogenet Genome Res 122:150-156

Ogawa A, Solovei I, Hutchison N et al (1997) Molecular characterization and cytological mapping of a non-repetitive DNA sequence region from the $\mathrm{W}$ chromosome of chicken and its use as a universal probe for sexing Carinatae birds. Chromosome Res 5:93-101

O'Neill RJ, Eldridge MD, Metcalfe CJ (2004) Centromere dynamics and chromosome evolution in marsupials. J Hered 95:375-381

Pevzner P, Tesler G (2003) Human and mouse genomic sequences reveal extensive breakpoint reuse in mammalian evolution. Proc Natl Acad Sci USA 100:7672-7677

Pichugin AM, Galkina SA, Potekhin AA, Punina EO, Rautian MS, Rodionov AV (2001) Determination of the minimum size of Gallus gallus domesticus chicken microchromosome by a pulse electrophoresis method. Genetika 37:657-660

Piras FM, Nergadze SG, Magnani E et al (2010) Uncoupling of satellite DNA and centromeric function in the genus Equus. PLoS Genet 6:e1000845

Rocchi M, Archidiacono N, Schempp W, Capozzi O, Stanyon R (2012) Centromere repositioning in mammals. Heredity 108:59-67 
Ruiz-Herrera A, Castresana J, Robinson TJ (2006) Is mammalian chromosomal evolution driven by regions of genome fragility? Genome Biol 7:R115

Ryttman H, Tegelstrom H (1981) G-banded karyotypes of three Galliformes species, domestic fowl (Gallus domesticus), quail (Coturnix coturnix japonica), and turkey (Meleagris gallopavo). Hereditas 94:165-170

Saifitdinova AF, Derjusheva SE, Malykh AG, Zhurov VG, Andreeva TF, Gaginskaya ER (2001) Centromeric tandem repeat from the chaffinch genome: isolation and molecular characterization. Genome 44:96-103

Sasaki M (1981) High resolution G-band karyotypes of the domestic fowl and the Japanese quail. Chrom Inform Service 31:26-28

Sasazaki S, Hinenoya T, Lin B, Fujiwara A, Mannen H (2006) A comparative map of macrochromosomes between chicken and Japanese quail based on orthologous genes. Anim Genet 37:316-320

Schmid M, Nanda I, Guttenbach M et al (2000) First report on chicken genes and chromosomes. Cytogenet Cell Genet 90:169-218

Schmid M, Nanda I, Hoehn H et al (2005) Second report on chicken genes and chromosomes. Cytogenet Genome Res 109:415-479

Shang WH, Hori T, Toyoda A et al (2010) Chickens possess centromeres with both extended tandem repeats and short non-tandem-repetitive sequences. Genome Res 20:12191228

Shibusawa M, Minai S, Nishida-Umehara C et al (2001) A comparative cytogenetic study of chromosome homology between chicken and Japanese quail. Cytogenet Cell Genet 95:103-109

Shibusawa M, Nishida-Umehara C, Masabanda J, Griffin DK, Isobe T, Matsuda Y (2002) Chromosome rearrangements between chicken and guinea fowl defined by comparative chromosome painting and FISH mapping of DNA clones. Cytogenet Genome Res 98:225-230

Shibusawa M, Nishibori M, Nishida-Umehara C et al (2004) Karyotypic evolution in the Galliformes: an examination of the process of karyotypic evolution by comparison of the molecular cytogenetic findings with the molecular phylogeny. Cytogenet Genome Res 106:111-119

Skinner BM, Griffin DK (2011) Intrachromosomal rearrangements in avian genome evolution: evidence for regions prone to breakpoints. Heredity 108:37-41
Skinner BM, Robertson LBW, Tempest HG et al (2009) Comparative genomics in chicken and Pekin duck using FISH mapping and microarray analysis. BMC Genomics 10:357

Slamovits CH, Rossi MS (2002) Satellite DNA: agent of chromosomal evolution in mammals. A review. J Neotrop Mammal 9:297-308

Solinhac R, Leroux S, Galkina S et al (2010) Integrative mapping analysis of chicken microchromosome 16 organization. BMC Genomics 11:616

Solovei IV, Joffe BI, Gaginskaya ER, Macgregor HC (1996) Transcription on lampbrush chromosomes of a centromerically localized highly repeated DNA in pigeon (Columba) relates to sequence arrangement. Chromosome Res 4:588-603

Solovei I, Ogawa A, Mitsuru N, Mizuno S, Macgregor H (1998) Specific chromomeres on the chicken W lampbrush chromosome contain specific repetitive DNA sequence families. Chromosome Res 6:323-327

Stock AD, Bunch TD (1982) The evolutionary implications of chromosome banding pattern homologies in the bird order Galliformes. Cytogenet Cell Genet 34:136-148

Tanaka K, Suzuki T, Nojiri T, Yamagata T, Namikawa T, Matsuda Y (2000) Characterization and chromosomal distribution of a novel satellite DNA sequence of Japanese quail (Coturnix coturnix japonica). J Hered 91:412-415

Telenius H, Pelmear AH, Tunnacliffe A et al (1992) Cytogenetic analysis by chromosome painting using DOP-PCR amplified flow-sorted chromosomes. Genes Chromosome Cancer 4:257-263

van Tuinen M, Dyke GJ (2004) Calibration of galliform molecular clocks using multiple fossils and genetic partitions. Mol Phylogenet Evol 30:74-86

Völker M, Backström N, Skinner BM et al (2010) Copy number variation, chromosome rearrangement, and their association with recombination during avian evolution. Genome Res 20:503-511

Wang X, Li J, Leung FC (2002) Partially inverted tandem repeat isolated from pericentric region of chicken chromosome 8 . Chromosome Res 10:73-82

Zhang Y, Zhang X, O'Hare TH et al (2011) A comparative physical map reveals the pattern of chromosomal evolution between the turkey (Meleagris gallopavo) and chicken (Gallus gallus) genomes. BMC Genomics 12:447

Zlotina A, Galkina S, Krasikova A et al (2010) Precise centromere positioning on chicken chromosome 3. Cytogenet Genome Res 129:310-313 